\title{
Combining FTIR microspectroscopy with differential scanning calorimetry
}

\author{
R.A. Spragg \\ Perkin Elmer Ltd., Post Office Lane, Beaconsfield, Bucks HP9 1QA, United Kingdom
}

The combination of FTIR spectroscopy with differential scanning calorimetry is potentially very powerful but little used. The problems of interfacing the two techniques are discussed, and a system using a novel DSC is described. The type of information obtainable with this combination is illustrated here with examples of phase changes in a liquid crystal, a synthetic polymer, and a crystalline organic compound. with any weight loss, such as crystallisation or glass transitions in polymers. IR spectroscopy provides qualitative information to complement the purely quantitative view provided by calorimetry. However, since a review in 1988 [1] there seems to be rather little reported work using the combination of FTIR and DSC. There have been many more articles in which FTIR is combined with thermogravimetric analysis (TGA). This article was prompted by the appearance of a very compact DSC instrument specifically designed for microspectroscopy [2]. Here we will discuss the practical issues involved in getting these techniques to work together, and the strengths and limitations of the combination.

\section{Practical considerations}

Differential scanning calorimetry (DSC) is a technique that measures heat flow changes as materials are heated or cooled. It can detect phase changes that are not associated
DSC measurements have to detect small transfers of heat into the sample, making it essential to minimise external 
thermal disturbances. In a conventional DSC, the sample is very well shielded from outside, and is packed to optimise heat transfer for what is a dynamic technique. The sample size is determined by a compromise. It has to be large enough to achieve a sizeable heat flow, but small enough to achieve rapid thermal equilibration. In practice the samples are typically several milligrams. On the other hand mid-IR measurements are quite demanding in terms of sample preparation. You need IR transmitting materials to provide a suitable light path. For transmission spectra the sample thickness is typically several tens of micrometers. There are two main issues in matching the two techniques. The DSC design has to allow access to IR without ruining the thermal performance. The sample size and presentation has to meet the needs of mid-IR which normally requires much smaller samples than DSC.

The main reason for using microspectroscopy is that it meets the need for thermal isolation of the sample by allowing the use of very small IR windows. In the case of powders, for example to study polymorphism, it makes it easier to find a region that is suitable for IR measurement.

A schematic of the DSC is shown in figure 1. It is a very compact system about $10 \mathrm{~cm}$ square by $2 \mathrm{~cm}$ thick that sits on the microscope stage. It has a separate controller box linked to a PC. It can be heated to $600{ }^{\circ} \mathrm{C}$ or cooled to liquid nitrogen temperature. The temperature can be programmed in a series of ramps at rates from 0.1 to tens of degrees a minute. Used as a DSC it measures the rate of heating required to maintain the temperature ramp. The device has a single sample pan so that a reference has to be run as a background in a separate measurement. The furnace can be purged, which is important as it avoids the problem of samples subliming on to the windows. The window materials can be chosen according to the spectral range of interest. Sapphire is commonly used for optical microscopy, but does not transmit beyond $1600 \mathrm{~cm}^{-1}$. Zinc sulphide would be suitable except that it precludes simultaneous visual examination of the sample. In practice $\mathrm{KBr}$ is probably the most widely useful window material.

For DSC measurements the sample is commonly sealed in an aluminium capsule. In this work the samples were contained in open pans of sapphire or aluminium. Others have used $\mathrm{KBr}$ windows with a retaining wall such as a metal ring. The aluminium pan can be used only to make reflection measurements. For weak absorptions the spectrum is essentially a transmission spectrum from radiation that has passed through the sample and been reflected from the base of the pan. If the sample absorbs strongly the spectrum is dominated by reflection from the top surface. The sample pans are considerably larger than the clear aperture available for transmission. With powders it is much easier to find a suitable area for measurement in reflection than in transmission because of the larger area available.

The most useful information is obtained by measuring spectra continuously as the temperature is changed. Time resolution is not critical as the rate of the temperature ramp can be varied. By triggering data collection at the start of

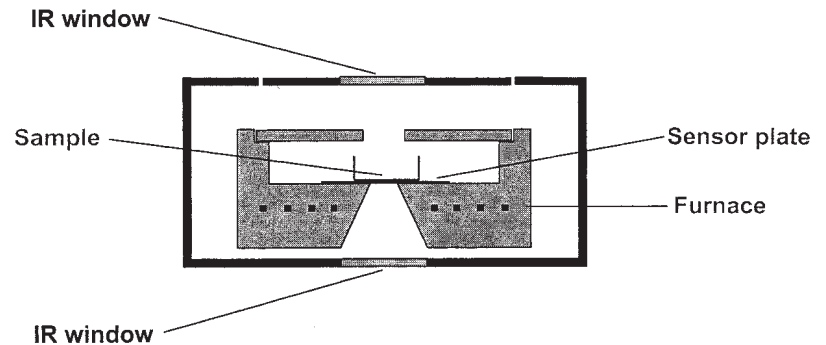

Figure 1. Schematic of the DSC.

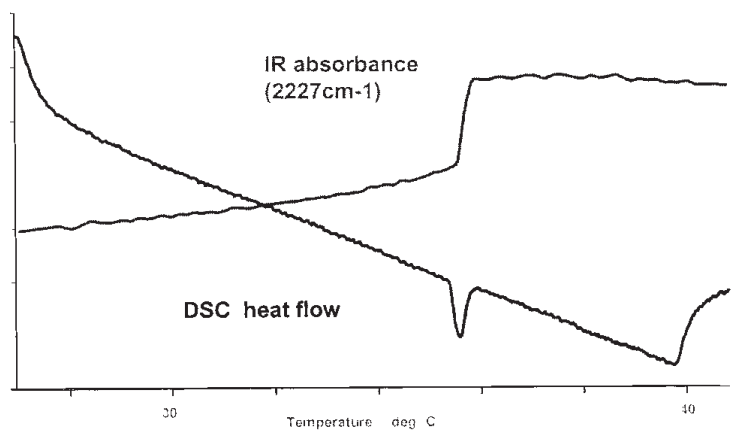

Figure 2. IR and DSC signals for 5CB nematic to isotropic transition.

the temperature ramp the temperature corresponding to each spectrum is known. The IR data can be examined in any of the ways used for other hyphenated measurements such as absorbance at specific frequencies, Gram-Schmidt, stacked or contour plots. All the data shown here were obtained using a Perkin Elmer AutoIMAGE microscope with a Spectrum BX FTIR spectrometer. Spectra were typically recorded with a $100 \times 100 \mu \mathrm{m}$ aperture at $4 \mathrm{~cm}^{-1}$ resolution.

\section{Applications}

Figure 2 shows data for a simple phase change in a liquid sample. $5 \mathrm{CB}$ is a liquid crystal that undergoes a nematic to isotropic phase change at around $35^{\circ} \mathrm{C}$. The molecule consists of an aromatic ring with a $\mathrm{CN}$ group and a long hydrocarbon chain. In the nematic phase there are domains of molecules that are aligned parallel to each other but this alignment is lost after the phase change. In the heat flow plot we see an absorption of energy in the form of a downward pointing peak. In the IR plot, based on the intensity of 


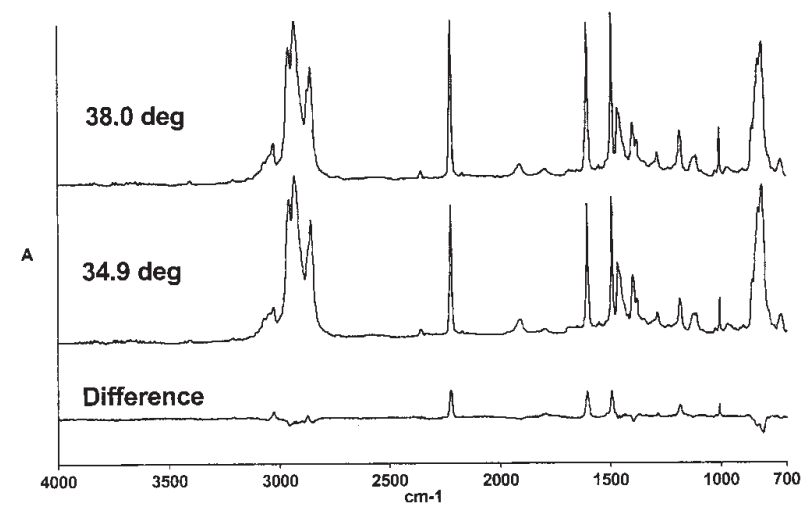

Figure 3. IR spectra of 5 CB above and below the nematic to isotropic transition.

the $\mathrm{CN}$ band at $2227 \mathrm{~cm}^{-1}$, we see a step change at the same temperature. In the nematic phase the region observed by IR has a preferred orientation. Because there is some intrinsic polarisation in the IR reflection measurement the intensity of the absorption is affected by the degree of orientation.

In figure 3 we can see that the phase change leads to increases in the intensities of the $\mathrm{CN}$ absorption and of some bands associated with the aromatic ring. Much larger changes would be observed if the measurements were with a polariser. As it is they serve to illustrate the high sensitivity of IR detection.

The practical problems can be seen by considering a classic DSC application relating to the recrystallisation of polyethylene terephthalate (PET). PET is widely used in bottles for pressurised beverages. It is critical to control the crystallinity of the PET and the standard method for measuring this is by DSC. When partially crystalline PET is heated the DSC curve shows a positive peak around 150 degrees as material recrystallises from what is essentially a supercooled amorphous phase, and then a negative peak as the material melts at about 250 degrees. The degree of crystallinity is measured from the ratio of the areas of these two peaks. Vibrational spectra can also be used to measure crystallinity but mid-IR measurements on PET are possible only on very thin films.

Figure 4 shows an IR spectrum of a PET sample in an aluminium cup. This was a $3 \mathrm{mg}$ sample cut from the lip of a PET bottle. The spectrum below $2000 \mathrm{~cm}^{-1}$ consists of specular reflection from the top surface. Above $2000 \mathrm{~cm}^{-1}$ it has the characteristics of a transmission spectrum as the weaker absorption allows radiation to pass through the sample and be reflected back from the base of the cup. In figure 5 are the IR and DSC signals obtained as the temperature was programmed at 10 degrees/minute from 40 to 320 degrees. The IR plot is of integrated absorbance using features above $3000 \mathrm{~cm}^{-1}$. The two curves show similar features although in the temperature range for recrystallisation

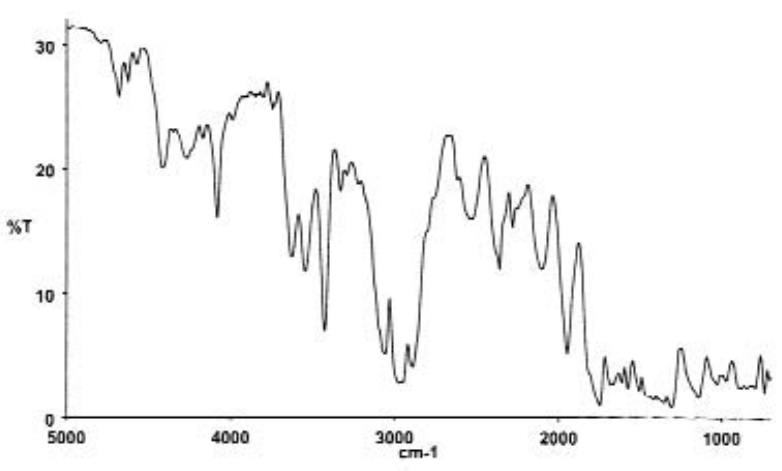

Figure 4. Spectrum of PET in aluminium cup.

the main IR changes appear to occur at lower temperature than the heat flow changes. The IR spectra in this temperature change show numerous small changes in band position and band shape. The band at $3440 \mathrm{~cm}^{-1}$ is typical. The width at half height changes from about $40 \mathrm{~cm}^{-1}$ to $34 \mathrm{~cm}^{-1}$ between 120 and $160^{\circ} \mathrm{C}$ (Fig. 6 and 7). The spectral changes seen are very similar to those in the mid-IR that are associated with changes in crystallinity.

The final example shows the transition of a crystal of methyl stearate from solid to liquid. The solid phase spectrum shows that the long $\mathrm{CH} 2$ chains adopt an extended zigzag conformation, producing the progression of bands seen between 1500 and $1000 \mathrm{~cm}^{-1}$. These are obviously absent in the liquid. For this the temperature was programmed at 1 degree/minute. The DSC heat flow shows that the transition around 40 degrees appears to have two stages, as can also be seen in the IR data. Examination of the IR spectra shows that the fine structure disappears over the temperature range $37.9-39.3{ }^{\circ} \mathrm{C}$, corresponding to the first

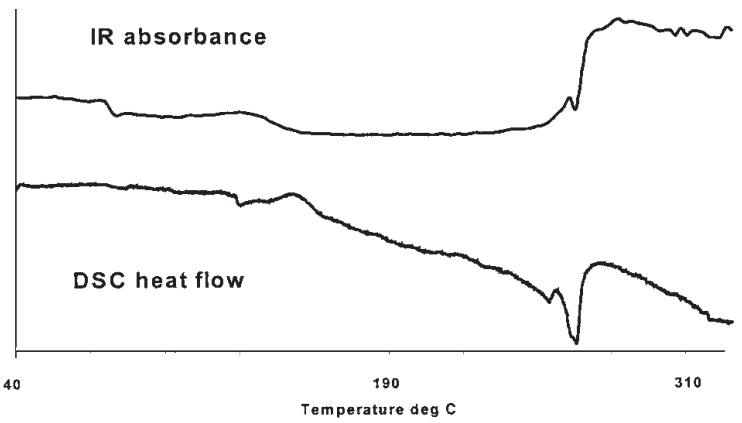

Figure 5. IR and DSC curves for PET. 


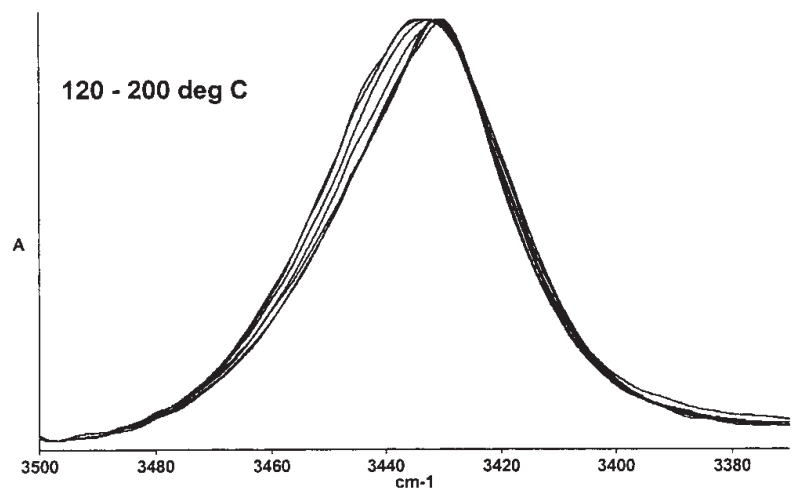

Figure 6. Band at $3440 \mathrm{~cm}^{-1}$, normalised intensity.

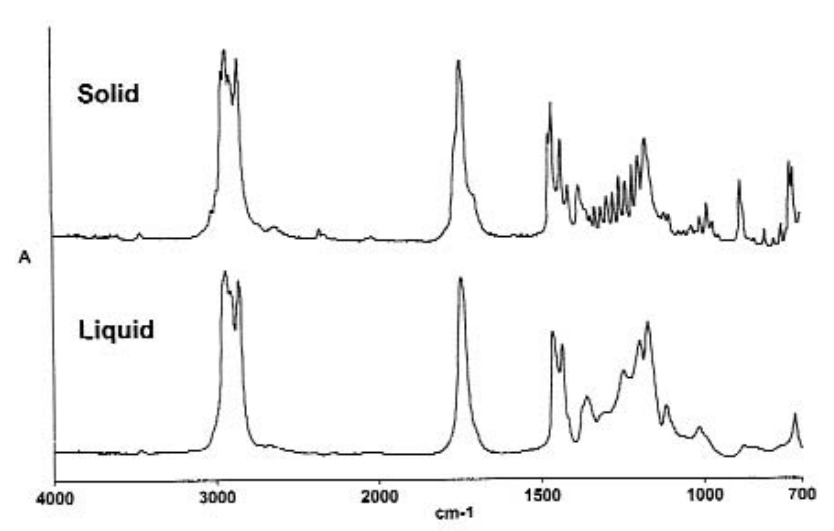

Figure 8. Spectra of methyl stearate.

peak in the DSC curve. There is little further change in this region, suggesting that the solid becomes amorphous before melting. The IR absorbance plot shows a continuing change at higher temperatures, but this simply results from the liquid sample spreading out after melting.

\section{Conclusions}

FTIR can clearly provide qualitative information that complements the heat flow changes measured by DSC. Interfacing the two techniques is very simple with a DSC

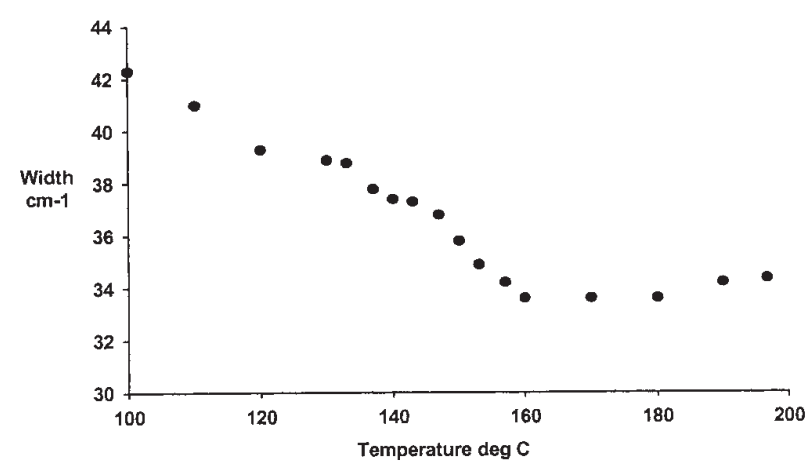

Figure 7 . Width at half height of band at $3440 \mathrm{~cm}^{-1}$.

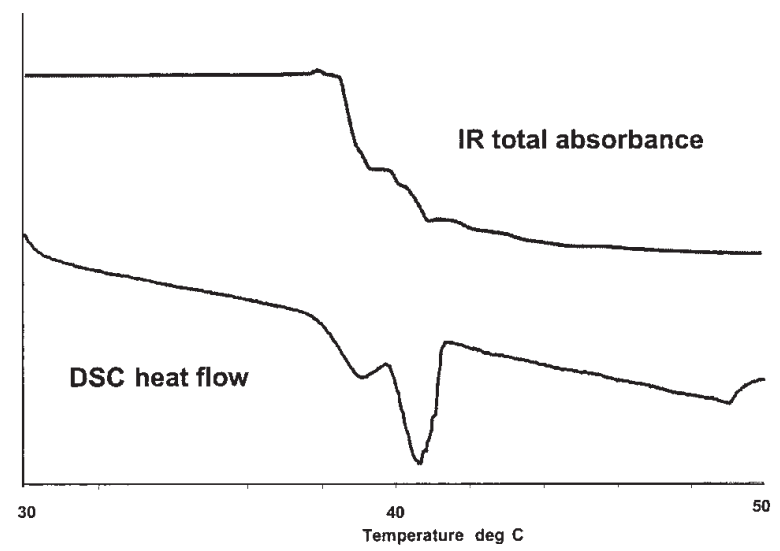

Figure 9. DSC and IR curves for methyl stearate.

system that mounts on a microscope stage. It is to be hoped that DSC-IR will become more widely recognised as a powerful tool for understanding thermal behaviour. The most promising fields of investigation are probably polymers and the polymorphism of pharmaceuticals.

\section{References}

1. Mirabella, F.M. in Infrared Microspectroscopy: Theory and Applications; Messerschmidt, R.G.; Harthcock, M.A. Ed., Marcel Dekker: New York, 1988, Ch. 5.

2. Linkam Scientific Instruments, Tadworth, Surrey, England. 